\begin{tabular}{lcc}
\hline Ročník LIII CCíslo 2, 2005 & 17
\end{tabular}

\title{
THE DAYTIME WOODY PLANTS WATER OUTPUT BY TRANSPIRATION
}

\author{
M. K. Akhmatov, P. Salaš
}

Received: March 30, 2004

\begin{abstract}
AKHMATOV, M. K., SALAŠ, P.: The daytime woody plants water output by transpiration. Acta univ. agric. et silvic. Mendel. Brun., 2005, LIII, No. 2, pp. 163-176

For the ecological-physiological research, and in particular water exchange, it is necessary to study a plant entire. The data about the daytime change of whole woody plant transpiration help to determine a degree of this or that woody species influence on air humidizing and that way on a microclimate as well, and also to receive necessary information about rational formation of woody plantings as the city boundaries and forest shelterbelts and on sites with various water-security.
\end{abstract}

woody plants, transpiration, microclimate, water output

For Kyrgyzstan, described by arid and semiarid climate, a heat control and air humidizing by vegetation plays an important role (especially in summertime) as the daytime temperature amplitude softening agent. In the large cities a number of jobs is responsible for the vegetation influence on a city microclimate. Kowashima [1990] has studied an influence of vegetative cover density on the Tokyo city parts and suburbs surface temperature and he has come to the conclusion that daytime temperature amplitudes in the city without gardening are insignificant, for planted trees and shrubs - is much higher, in suburb the return picture is observed; at night in the planted most trees and shrubs places of city lower temperatures, in the planted most trees and shrubs suburbs - higher are observed. Researches [Wilmers, 1990] of microclimate and structure of vegetation poleochoroclimatops, for example in Hannover, have shown the vegetation plays an important role as the temperature amplitude softening agent and power of this influences depends on cover density and evapotranspiration intensity. Saito, Ishihara and Kalayama [1990] have evolved detailed study of relations between vegetation distribution and meteorological elements in Kumamoto city and they have obtained data about the temperature difference between urban and suburban zones. This difference has made $3{ }^{\circ} \mathrm{C}$ in daytime and $2{ }^{\circ} \mathrm{C}$ at night. Between the temperatures inside of the city park and behind its borders the difference in $3{ }^{\circ} \mathrm{C}$ was observed. For Hyderabad city [Raza et al., 1990] it is shown, that the role of trees for the city climate maintenance depends in many cases on the form and size of a woody plant crown and also on the leaf morphology.

There was also an investigation about the green plantings influence on the air temperature in cities of the former USSR. The data received in Moscow [Anastasyev, Kharakhinov, 1936; Mashinsky, 1962] and Tashkent [Chistyakova, Semenova; 1962] have shown, that the temperature of air among buildings in all cases appeared above the air temperature among green plantings, and the difference of these temperatures sometimes reached 10-12\%. Izdebsky [1949] has proved that more favourable temperature mode is created among plantings in the winter; the temperature at landscaping areas is higher than on open space areas. Experiments carried out by Kuzmina [1970] in Frunze (Bishkek) have showed that various wood plant species do not give the identical shading area (in $\%$ of the general area 
under a tree crown), but the shading area can range from $50 \%$ at Caragana boisii Schneid. up to $97 \%$ at Ulmus pinnato-ramosa Dieck. ex Kochne. An urban plantings also influence humidity of air. The relative air humidity measurement, made by Anastasyeva and Kharakhinova [1936], shown $27 \%$ and $16 \%$ increasing of air humidity in the city park and adjacent park pathways compare to the square. Lunts [1974] has considered increase of relative air humidity is by the persons generally perceived as temperature downturn; increasing of relative air humidity for example of $15 \%$ is by human body perceived as air temperature downturn of $3.5^{\circ} \mathrm{C}$.

Intensity of transpiration is usually measured in milligrams or grams per square decimeters or grams of crude weight and one hour daytime dynamics. According to Libbert [1976] it is possible to get the data that one birch transpiration water loss can rich up to 4001 per day and beech planting returns by transpiration into the atmosphere about $60 \%$ of its watering deposit. Villi and Detje [1974] have specified the average size trees ability of transpiration at more than 2001 of water per day. One hectare of corn can for transpiration use 35000001 of water during the vegetative period and 1 hectare of mature maple wood approximately twice more than it. Compare to this data field the cactuses in Arizona Desert can spend no more than 27501 of water per 1 hectare within the whole year. Nesterov [1949] has established that 1 woods hectare can evaporate into the atmosphere about 1-3.5 million kilograms of moisture per year; that makes from $20 \%$ to $70 \%$ of an atmospheric precipitation. According to Popov and Jakovleva [1996], a 17-year old poplar growing along the channel, can evaporate $73 \mathrm{~m}^{3}$; an oak (Quercus imbricaria Michx.) $-20 \mathrm{~m}^{3}$ and an ash (Fraxinus viridis $)-13 \mathrm{~m}^{3}$ of water.

As the purpose of present researches it is studying of the daytime water consumption for the need of whole wood plant transpiration and the definition of chosen woody plants influence on the microclimate and its humidifying.

\section{MATERIAL AND METHODS}

As the object of this research were chosen deciduous wood plants of three vital forms, introduced into the Botanical garden of the Kyrgyz Republic National Academy of sciences. 15 kinds of trees - Aesculus hippocastanum L., Cercis canadensis L., Betula procurva Litv., Acer saccharinum L., Acer platanoides L., Acer pseudoplatanus L., Quercus robur L., Quercus imbricaria Michx., Sorbus intermedia (Ehrh.) Pers., Juglans regia L., Carpinus betulus L., Ulmus pinnato-ramosa Dieck. ex Kochne., Padus racemosa (L.). Gillib., Populus nigra L. 'Italica', Crataegus altaica Lge.; 16 kinds of bushes - Caragana boisii Schneid., Philadelphus lewisii Pursh., Elaeagnus angustifolia L., Euonymus maackii Rupr., Forsythia suspensa (Thunb.) Vahl., Viburnum lantana L., Rhus typhina L., Spiraea $\times$ vanhouttei (Briol.) Zab., Chaenomeles japonica (Thunb.) Lind., Berberis oblonga (Regel) Schneid., Ligustrum vulgare L., Symphoricarpos albus (L). Blake., Cornus sanguinea L., Syringa amurensis Rupr., Spiraea lasiocarpa Kar. et. Kir., Cotinus coggygria Scop.; and 1 kind of liana - Wisteria floribunda (Willd.) DC.

Intensity of transpiration has been determined by the field transpirometers in accordance with Shpota's method [1992] with (Akhmatov, Oskonbaeva, Shpota, 1999) modification in a design of transpirometers and in a technique of its use on many objects. For the studying of the daytime water consumption for the need of whole wood plant transpiration, the area of separate leaves and also the general leaf surface during vegetation have been simultaneously determined according the Kochkumbayev at al. [1999] method. On the basis of received data a curve of change dynamics of the common leaf surface has been built and the amount of leaf-days $\left(\mathrm{m}^{2}\right.$.day $\left.{ }^{-1}\right)$ has been counted up. After that, using the data about the intensity of transpiration, the daytime water consumption for the transpiration has been determined. Results have been statistically analysed by methods of Dospechov (1979) and Shpota (1992). The results are stated in Table VI.-X.

\section{RESULTS AND DISCUSSION}

The calculations of the daytime water consumption for the need of whole wood plant transpiration in 1998 are submitted in Table I. The experiment results have shown that the lowest parameters of transpiration per day are presented in May when the tree leaf surface just starts to be formed. However at one species, Acer saccharinum L., the high values $(425,0 \mathrm{~kg})$ of the daytime water consumption for transpiration have been already observed at this time. Later on, during process of leaf area increasing, the amount of water transpired from the total leaf surface of tree grows. In September, at the beginning of leaf abscission, the woody plants reduce weight of evaporated moisture. Close to the end of leaf biomass increasing, the daytime water consumption for transpiration has continued to change at all investigated tree species, which is connected to parameters of transpiration intensity in various days. As Populus nigra L. 'Italica' . differs itself with its high values of transpiration intensity and the general leaf surface, also the amount of its evaporated water per day in separate days can exceed $2000 \mathrm{~kg}$.

Other examples, Aesculus hippocastanum L. and Cercis canadensis L., described by huge total leaf 
area and, on the other hand, also by low transpiration intensity, have reached in daytime water consumption for transpiration values not exceed $412.5 \mathrm{~kg}$, respectively $276.0 \mathrm{~kg}$. More than $1000 \mathrm{~kg}$ per day, it is the evaporation capacity of Acer saccharinum L.; this species, together with Populus nigra L. 'Italica', Acer pseudoplatanus L., Acer platanoides L., Quercus robur L., Quercus imbricaria Mtchx. and
Ulmus pinnato-ramosa Dieck. ex Kochne., is possible to class in the species having average indices of water evaporated per day, which reaches a level of $500 \mathrm{~kg}$ and more. The least amount of water is transpired by Crataegus altaica Lge. (maximally $165.3 \mathrm{~kg}$ per day), distinguished by high intensity of transpiration and low value of total leaf surface.

I: The daytime water consumption for the need of whole tree transpiration in $1998\left({\left.\mathrm{~kg} . d a y^{-1}\right)}^{-1}\right.$

\begin{tabular}{|l|r|r|r|r|r|r|r|r|}
\hline \multirow{2}{*}{ Species } & \multicolumn{9}{c|}{ Date } \\
\cline { 2 - 9 } & $\mathbf{1 4 . 5 .}$ & $\mathbf{2 7 . 5 .}$ & $\mathbf{9 . 6 .}$ & $\mathbf{2 6 . 6 .}$ & $\mathbf{9 . 7 .}$ & $\mathbf{2 7 . 7 .}$ & \multicolumn{1}{c|}{$\mathbf{1 0 . 8 .}$} & $\mathbf{7 . 9 .}$ \\
\hline Populus nigra 'Italica' & 121.8 & 1369.0 & 660.0 & 1952.5 & 2007.5 & 1870.0 & 1787.5 & 990.0 \\
\hline Crataegus altaica & 44.8 & 165.3 & 144.0 & 120.0 & 124.0 & 83.20 & 45.0 & 23.8 \\
\hline Juglans regia & - & 219.2 & 240.0 & 244.4 & 192.0 & 235.2 & 220.8 & 171.2 \\
\hline Quercus robur & 81.0 & 210.0 & 223.2 & 416.0 & 410.8 & 572.0 & 384.8 & 283.4 \\
\hline Quercus imbricaria & - & 126.0 & 332.8 & 326.8 & 559.0 & 602.0 & 559.0 & 365.5 \\
\hline Acer pseudoplatanus & 67.2 & 253.0 & 336.0 & 468.0 & 456.0 & 448.5 & 513.5 & 143.0 \\
\hline Acer platanoides & 150.4 & 336.0 & 323.2 & 598.4 & 550.4 & 528.0 & 646.4 & 352.0 \\
\hline Acer saccharinum & 425.0 & 542.5 & 794.5 & 1050.0 & 682.5 & 1155.0 & 1137.5 & 542.5 \\
\hline Carpinus betulus & 50.5 & 112.0 & 142.0 & 253.8 & 255.3 & 194.0 & 240.5 & 160.0 \\
\hline Ulmus pinnato-ramosa & 49.5 & 175.5 & 176.0 & 219.2 & 320.0 & 448.0 & 240.0 & 208.0 \\
\hline Sorbus intermedia & 72.0 & 172.8 & 186.0 & 240.0 & 216.0 & 196.8 & 240.0 & 168.0 \\
\hline Aesculus hippocastanum & 178.2 & 373.0 & 376.2 & 442.2 & 412.5 & 297.0 & 330.0 & 237.6 \\
\hline Cercis canadensis & - & 110.0 & 150.0 & 170.0 & 184.0 & 230.0 & 276.0 & 198.0 \\
\hline Padus racemosa & 81.6 & 182.0 & 221.2 & 240.8 & 168.0 & 210.0 & 238.0 & 144.2 \\
\hline Betula procurva & 138.6 & 391.6 & 363.0 & 374.0 & 275.0 & 323.4 & 374.0 & 165.0 \\
\hline
\end{tabular}

The data of the daytime water consumption for whole trees transpiration at the rate of years 1999 and 2000 are submitted in Table II. The results have showed that Populus nigra L. 'Italica', a champion in transpiration, has used for transpiration up to $1935.0 \mathrm{~kg}$, respectively $2832.5 \mathrm{~kg}$ of water per day. From 462.0 up to $834.9 \mathrm{~kg}$ of water per day can evaporate leaves of Acer saccharinum L. An average positions between indications of transpiration water consumption is occupied by Acer pseudoplatanus L., Acer platanoides L., Quercus robur L., Quercus imbricaria Michx. and Aesculus hippocastanum L. Less amount of water is transpired by Padus racemosa (L.) Gillib. and Crataegus altaica Lge. 191.8 and $172.8 \mathrm{~kg}$ per day. 
II: The daytime water consumption for whole tree transpiration in 1999-2000 (kg.day $\left.{ }^{-1}\right)$

\begin{tabular}{|c|c|c|c|c|c|c|}
\hline \multirow{3}{*}{ Species } & \multicolumn{6}{|c|}{ Date } \\
\hline & \multicolumn{3}{|c|}{1999} & \multicolumn{3}{|c|}{2000} \\
\hline & 19.6. & 25.7 . & 9.8. & 11.6. & 19. 7. & 7.8. \\
\hline Crataegus altaica & 148.5 & 135.0 & 121.5 & 172.8 & 144.4 & 124.2 \\
\hline Populus nigra 'Italica' & 2832.5 & 1045.0 & 1815.0 & 1935.0 & 1598.4 & 1047.6 \\
\hline Juglans regia & 342.0 & 247.0 & 190.0 & 294.0 & 217.0 & 215.2 \\
\hline Quercus robur & 211.2 & 297.0 & 379.5 & 291.0 & 390.0 & 375.0 \\
\hline Quercus imbricaria & 303.4 & 389.5 & 328.0 & 466.4 & 422.4 & 369.6 \\
\hline Acer pseudoplatanus & 179.4 & 227.7 & 350.0 & 309.0 & 402.0 & 204.0 \\
\hline Acer platanoides & 240.0 & 280.0 & 299.0 & 414.4 & 499.5 & 518.0 \\
\hline Acer saccharinum & 693.0 & 462.0 & 561.0 & 616.0 & 547.8 & 834.9 \\
\hline Carpinus betulus & 168.0 & 159.6 & 152.0 & 166.5 & 219.8 & 111.6 \\
\hline Ulmus pinnato-ramosa & 240.0 & 202.5 & 195.0 & 210.0 & 252.0 & 232.5 \\
\hline Sorbus intermedia & 245.0 & 248.5 & 168.0 & 264.0 & 282.4 & 254.4 \\
\hline Aesculus hippocastanum & 297.0 & 126.0 & 129.5 & 370.0 & 273.8 & 251.6 \\
\hline Cercis canadensis & 225.0 & 150.0 & 300.0 & 130.0 & 156.0 & 286.0 \\
\hline Padus racemosa & 108.0 & 148.0 & 144.0 & 171.3 & 112.3 & 191.8 \\
\hline Betula procurva & 265.0 & 340.0 & 400.0 & 224.0 & 302.4 & 409.5 \\
\hline
\end{tabular}

The three-year data about the daytime trees consumption of water for transpiration have testify that in the greater degree it depends on its total leaf surface though also values of transpiration intensity influence the final result. Weak transpiration intensity of some tree species seems to be the main factor influencing the water amount evaporated per day. The received data can informed us about the influence of monitored tree species on air humidifying and partially also on microclimate.

The data about daytime whole bushes and liana water consumption for transpiration in 1997 are submitted in the Table III. A significant amount of water (from 154.0 up to $364.0 \mathrm{~kg}$.day-a) can be evaporated by Cotinus coggygria Scop. and this makes it the bush with the highest intensity of transpiration. Rhus typhina L., Viburnum lantana L. and Philadelphus lewisii Pursh. can transpire more than $100 \mathrm{~kg}$ of water per day, though the first one due to the greater total leaf surface, and next two due to their high intensity of transpiration. Sufficient amount of water is evaporated also by Elaeagnus angustifolia L. (20.0-91.8 kg.day $\left.{ }^{-1}\right)$, Forsythia suspensa (Thunb.) Vahl. (36.0-59.4 kg.day $\left.{ }^{-1}\right)$ and Spiraea $\times$ vanhouttei (Briot.) Zab. (33.3-97.2 kg.day $\left.{ }^{-1}\right)$. In certain days Wisteria floribunda (Willd.) DC. and Caragana boisii Schneid. have evaporate more than $30 \mathrm{~kg}$ of water per day. The least amount of water has been evaporated by Ligustrum vulgare L. (1.7-3.7 kg.day $\left.{ }^{-1}\right)$ and
Cornus sanguinea L. (2.3-15.6 kg.day $\left.{ }^{-1}\right)$. Other kinds of bushes have occupied the places in between.

The data about daytime water consumption for transpiration of bushes and liana in 1998 are submitted in Table IV. From all of the studied bushes, the largest amount of transpired water has been reached by Cotinus coggygria Scop. So, on August 12 , the daytime water consumption for transpiration has got $220 \mathrm{~kg}$ day $^{-1}$, and in other evaluated days it has exceeded $100 \mathrm{~kg}$ of water per day. In addition to this bush species, more than $100 \mathrm{~kg}$ of water per day were transpired also by Viburnum lantana L. and Philadelphus lewisii Pursh. There were observed days, when such bushes as Rhus typhina L. and Elaeagnus angustifolia L. had also an opportunity to evaporate significant water amount by their leaf surface, namely 86.6 , respectively $68.6 \mathrm{~kg}$.day ${ }^{-1}$. By average indices the species Syringa amurensis Rupr., Forsythia suspensa (Thunb.) Vahl., Spiraea $\times$ vanhouttei (Briol.) Zab. and Caragana boisii Schneid. have been characterized. Because of the small total leaf surfaces and weak intensity of transpiration, a small amount of water was transpired by Ligustrum vulgare L. and Cornus sanquinea L. which can be expressed by values $1.8-6.2{\mathrm{~kg} . \mathrm{day}^{-1}}^{-1}$ and $2.2-9.9 \mathrm{~kg}^{- \text {day }^{-1}}$ respectively.

In 1999 the evaluation of the daytime bushes and liana water consumption for transpiration, calculated from the total leaf surface (Table V.) has been 
continued. As a whole, the data received from this year have confirmed previous results.

Cotinus coggygria Scop. is distinguished by the high values of water evaporated per day - 133.0 $179.2 \mathrm{~kg}$. In the end of June Viburnum lantana L. and Philadelphus lewisii Pursh. have transpired by the total area of leaves more water than $100 \mathrm{~kg}^{\text {day }}{ }^{-1}$ though there were also days when their daytime water consumption for transpiration was reduced to 49.2 and $56.7 \mathrm{~kg}$.day ${ }^{-1}$, respectively. More than $20 \mathrm{~kg} \cdot \mathrm{day}^{-1}$ has been used for the daytime transpiration by such bushes, as Rhus typhina L., Syringa amurensis Rupr., Forsythia suspensa (Thunb.) Vahl., Spiraea $\times$ vanhouttei (Briot.) Zab. and Caragana boisii Schneid. For some of them, the amount of water used for transpiring within day can achieve big values. For example, Forsythia suspensa (Thunb.) Vahl. can use water at the volume $96.2 \mathrm{~kg} \cdot \mathrm{day}^{-1}$, Rhus typhina L. $63.5 \mathrm{~kg}$.day ${ }^{-1}$ and Spiraea $\times$ vanhouttei (Briot).

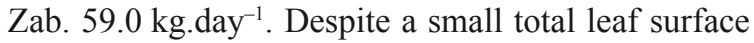
the species Euonymus maackii Rupr., Chaenomeles japonica (Thunb.) Lindl. and Symphoricarpos albus (L). Blake. have reached the high values of transpiration intensity - they can evaporate more water than $10 \mathrm{~kg}$.day ${ }^{-1}$. The Elaeagnus angustifolia L. data are unbalanced and its disorder is rather significant - from 12.0 up to $50.0 \mathrm{~kg}$ of water per day. The least daytime water consumption for transpiration has been made by Ligustrum vulgare L. - 2.7$4.7 \mathrm{~kg}$. day ${ }^{-1}$. Less transpiration from the total leaf surface has been observed also at Cornus sanquinea L. and Chaenomeles japonica (Thunb.) Lindl. A liana Wisteria floribunda (Willd.) DC. has transpired up to $15.0-18.5 \mathrm{~kg}$ of water per day.

III: Daytime water consumption for transpiration of bushes and liana in 1997 (kg.day $\left.{ }^{-1}\right)$

\begin{tabular}{|l|r|r|r|r|r|r|}
\hline \multirow{2}{*}{ Species } & \multicolumn{7}{c|}{ Date } \\
\cline { 2 - 7 } & $\mathbf{1 7 . 6 .}$ & $\mathbf{2 7 . 6 .}$ & \multicolumn{1}{|c|}{$\mathbf{1 1 . 7}$} & \multicolumn{1}{|c|}{$\mathbf{7 4 .}$} & \multicolumn{1}{|c|}{$\mathbf{8 .}$} & $\mathbf{2 0 . 8 .}$ \\
\hline Rhus typhina & 140.25 & 129.25 & 200.75 & 145.75 & 108.65 & 27.60 \\
\hline Syringa amurensis & 16.87 & 17.01 & 20.35 & 24.96 & 19.50 & 15.90 \\
\hline Elaeagnus angustifolia & 55.46 & 38.40 & 21.90 & 20.00 & 91.80 & 91.30 \\
\hline Philadelphus lewisii & 123.25 & 194.30 & 137.75 & 145.00 & 137.70 & 137.00 \\
\hline Forsythia suspensa & 40.00 & 54.45 & 59.40 & 57.00 & 47.50 & 36.00 \\
\hline Euonymus maackii & 18.70 & 29.48 & 20.90 & 22.00 & 20.90 & 18.26 \\
\hline Wisteria floribunda & 36.25 & 13.75 & 26.25 & 38.75 & 16.25 & 17.50 \\
\hline Viburnum lantana & 41.00 & 13.00 & 177.00 & 133.50 & 87.50 & 100.00 \\
\hline Chaenomeles japonica & 10.76 & 11.87 & 20.00 & 17.00 & 14.75 & 15.25 \\
\hline Symphoricarpus albus & 32.60 & 27.60 & 29.40 & 14.96 & 16.00 & 9.90 \\
\hline Ligustrum vulgare & 2.76 & 1.74 & 2.80 & 0.80 & 3.65 & 1.45 \\
\hline Cornus sanguinea & 7.83 & 7.56 & 15.66 & 2.25 & 9.00 & 7.18 \\
\hline Berberis oblonga & 14.50 & 7.25 & 7.25 & 8.50 & 9.25 & 13.75 \\
\hline Cotinus coggygria & 154.00 & 245.00 & 311.50 & 262.50 & 364.00 & 322.00 \\
\hline Spiraea $\times$ vanhouttei & 83.70 & 70.20 & 67.20 & 33.30 & 65.40 & 64.60 \\
\hline Caragana boisii & 14.00 & 31.50 & 32.60 & 27.70 & 23.70 & 18.60 \\
\hline Spiraea lasiocarpa & 10.00 & 12.60 & 19.20 & 20.00 & 13.80 & 10.20 \\
\hline
\end{tabular}


IV: The day time water consumption for transpiration of bushes and liana in $1998\left(\mathrm{~kg}^{\left.-d a y^{-1}\right)}\right.$

\begin{tabular}{|l|r|r|r|r|r|r|}
\hline \multirow{2}{*}{ Species } & \multicolumn{7}{c|}{ Date } \\
\cline { 2 - 7 } & $\mathbf{1 5 . 5 .}$ & $\mathbf{1 2 . 6 .}$ & $\mathbf{2 9 . 7 .}$ & $\mathbf{1 2 . 8 .}$ & $\mathbf{1 6 . 9 .}$ & \multicolumn{1}{c|}{$\mathbf{2 0 . 8 .}$} \\
\hline Rhus typhina & \multicolumn{1}{|c|}{} & 84.60 & 86.60 & 70.90 & 38.32 & 32.43 \\
\hline Syringa amurensis & 12.60 & 42.90 & 19.80 & 44.55 & 45.21 & 18.00 \\
\hline Elaeagnus angustifolia & 40.80 & 68.64 & 36.90 & 16.30 & 28.80 & 8.60 \\
\hline Philadelphus lewisii & 36.25 & 90.00 & 103.50 & 88.20 & 95.10 & 42.00 \\
\hline Forsythia suspensa & 16.50 & 36.75 & 26.25 & 47.25 & 56.70 & 20.80 \\
\hline Euonymus maackii & 5.27 & 15.58 & 17.60 & 16.28 & 11.44 & 6.20 \\
\hline Wisteria floribunda & - & 10.00 & 16.25 & 19.25 & 20.00 & 17.00 \\
\hline Viburnum lantana & 45.50 & 112.20 & 75.00 & 39.00 & 78.00 & 38.00 \\
\hline Chaenomeles japonica & 5.51 & 11.37 & 13.44 & 9.24 & 13.16 & 6.17 \\
\hline Symphoricarpos albus & 12.47 & 35.90 & 16.32 & 14.20 & 13.63 & 6.00 \\
\hline Ligustrum vulgare & 1.80 & 5.32 & 5.60 & 4.92 & 6.20 & 2.80 \\
\hline Cornus sanquinea & 3.80 & 7.95 & 9.90 & 8.25 & 8.16 & 2.22 \\
\hline Berberis oblonga & 3.10 & 12.50 & 9.00 & 7.75 & 7.10 & 7.40 \\
\hline Cotinus coggygria & - & 136.80 & 216.00 & 192.00 & 220.00 & 132.00 \\
\hline Spiraea $\times$ vanhouttei & 16.00 & 34.00 & 38.00 & 30.00 & 30.00 & 9.00 \\
\hline Caragana boisii & 16.00 & 32.00 & 36.00 & 40.00 & 42.00 & 24.60 \\
\hline Spiraea lasiocarpa & 3.52 & 8.50 & 11.80 & 9.60 & 11.60 & 4.20 \\
\hline
\end{tabular}

V: The daytime bush and liana water consumption for transpiration in 1999 (kg.day-1)

\begin{tabular}{|l|r|r|r|r|}
\hline \multirow{2}{*}{ Species } & \multicolumn{4}{c|}{ Date } \\
\cline { 2 - 5 } & $\mathbf{2 1 . 6 .}$ & $\mathbf{2 6 . 7 .}$ & \multicolumn{1}{c|}{$\mathbf{1 8 . 8 .}$} & \multicolumn{1}{c|}{$\mathbf{1 2 . 9 .}$} \\
\hline Rhus typhina & 63.45 & 63.45 & 23.50 & 27.90 \\
\hline Syringa amurensis & 26.55 & 39.00 & 45.00 & 25.98 \\
\hline Elaeagnus angustifolia & 14.00 & 12.00 & 50.00 & 12.00 \\
\hline Philadelphus lewisii & 117.00 & 92.80 & 88.00 & 56.70 \\
\hline Forsythia suspensa & 25.60 & 39.20 & 96.20 & 31.50 \\
\hline Euonymus maackii & 18.00 & 15.50 & 11.85 & 9.58 \\
\hline Wisteria floribunda & 16.25 & 18.50 & 15.00 & 16.00 \\
\hline Viburnum lantana & 102.00 & 49.20 & 78.00 & 58.80 \\
\hline Chaenomeles japonica & 9.60 & 7.90 & 9.37 & 8.00 \\
\hline Symphoricarpos albus & 16.38 & 7.78 & 7.80 & 6.20 \\
\hline Ligustrum vulgare & 4.40 & 3.44 & 4.72 & 2.72 \\
\hline Cornus sanquinea & 6.90 & 5.16 & 8.10 & 3.20 \\
\hline Berberis oblonga & 9.13 & 6.30 & 9.57 & 6.75 \\
\hline Cotinus coggygria & 133.00 & 157.50 & 179.20 & 145.60 \\
\hline Spiraea $\times$ vanhouttei & 34.40 & 45.00 & 59.00 & 27.00 \\
\hline Caragana boisii & 26.00 & 44.00 & 35.00 & 28.00 \\
\hline Spiraea lasiocarpa & 11.50 & 15.00 & 14.50 & 8.20 \\
\hline
\end{tabular}




\begin{tabular}{|c|c|c|c|c|c|c|c|c|c|c|c|c|c|c|c|}
\hline$\stackrel{0}{\circ}$ & 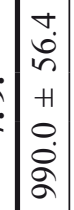 & 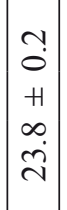 & 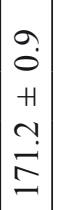 & 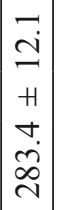 & $\mid \begin{array}{l}n \\
\alpha \\
+1 \\
n \\
n \\
b \\
n \\
n\end{array}$ & 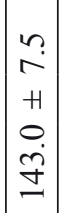 & $\begin{array}{c}n \\
n \\
+1 \\
0 \\
0 \\
\tilde{n} \\
n \\
n\end{array}$ & 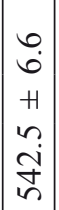 & $\begin{array}{l}\hat{i} \\
+ \\
\dot{0} \\
\ddot{\theta} \\
\ddot{\theta}\end{array}$ & $\begin{array}{c}-\vec{r} \\
+ \\
+ \\
0 \\
\infty \\
\stackrel{\sim}{\vec{d}}\end{array}$ & \begin{tabular}{|c|}
$\tilde{\gamma}$ \\
+ \\
$H$ \\
0 \\
0 \\
0 \\
0
\end{tabular} & $\mid \begin{array}{c}a \\
\vec{i} \\
+1 \\
0 \\
\hat{v} \\
\vec{v}\end{array}$ & $\begin{array}{l}a \\
\dot{r} \\
+ \\
\dot{0} \\
\dot{\infty} \\
\varrho\end{array}$ & 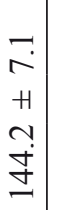 & $\begin{array}{l}\infty \\
i \\
+1 \\
0 \\
0 \\
\dot{b} \\
0\end{array}$ \\
\hline $\begin{array}{l}\infty \\
\dot{\theta} \\
\dot{0}\end{array}$ & $\mid \begin{array}{c}a \\
\Sigma \\
= \\
+1 \\
n \\
\infty \\
\infty \\
\Sigma\end{array}$ & \begin{tabular}{|c|}
0 \\
$\dot{0}$ \\
+ \\
0 \\
$\dot{j}$ \\
$\dot{\sigma}$
\end{tabular} & 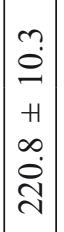 & $\begin{array}{l}- \\
\vec{\Xi} \\
+ \\
\infty \\
\infty \\
\dot{+} \\
m\end{array}$ & $\begin{array}{l}\hat{i} \\
\tilde{n} \\
+ \\
\dot{a} \\
\dot{\hat{n}} \\
\hat{n}\end{array}$ & $\mid \begin{array}{l}a \\
\hat{n} \\
+1 \\
n \\
n \\
\tilde{n} \\
\bar{n}\end{array}$ & $\begin{array}{l}2 \\
i \\
+1 \\
t \\
\dot{0} \\
\dot{0}\end{array}$ & $\begin{array}{c}0 \\
\infty \\
+1 \\
n \\
\tilde{n} \\
= \\
=\end{array}$ & $\begin{array}{l}0 \\
\dot{r} \\
+1 \\
n \\
\tilde{+} \\
\stackrel{v}{v}\end{array}$ & $\mid \begin{array}{c}\infty \\
\dot{0} \\
+1 \\
0 \\
\dot{0} \\
\dot{j} \\
\sim\end{array}$ & 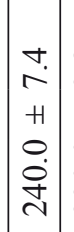 & $\begin{array}{c}\infty \\
\infty \\
\dot{n} \\
+1 \\
0 \\
\dot{0} \\
\tilde{m}\end{array}$ & 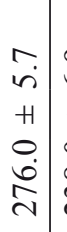 & 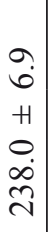 & $\begin{array}{l}n \\
n \\
+1 \\
0 \\
\dot{+} \\
\text { nn }\end{array}$ \\
\hline & $\mid \begin{array}{c}n \\
0 \\
i \\
+1 \\
+ \\
0 \\
\dot{0} \\
\infty \\
0\end{array}$ & 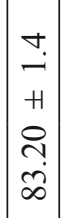 & $\mid \begin{array}{c}0 \\
a \\
+1 \\
\sim \\
\tilde{n} \\
\tilde{\sim}\end{array}$ & $\begin{array}{l}= \\
\dot{I} \\
+ \\
0 \\
\stackrel{i}{i} \\
i n\end{array}$ & $\begin{array}{l}\overrightarrow{\dot{d}} \\
\dot{n} \\
+ \\
0 \\
\dot{\delta} \\
\dot{\delta}\end{array}$ & $\mid \begin{array}{c}0 \\
\dot{d} \\
\tilde{n} \\
+ \\
n \\
n \\
\dot{0} \\
\dot{f}\end{array}$ & $\begin{array}{c}0 \\
\dot{n} \\
+1 \\
0 \\
0 \\
\infty \\
\tilde{n} \\
\tilde{n}\end{array}$ & $\begin{array}{c}m \\
\infty \\
+1 \\
0 \\
\dot{n} \\
\underline{n} \\
=\end{array}$ & $\begin{array}{l}n \\
\dot{+} \\
+ \\
0 \\
\dot{+} \\
\stackrel{\sigma}{-}\end{array}$ & $\begin{array}{c}0 \\
\dot{0} \\
+1 \\
0 \\
\dot{0} \\
\dot{0} \\
\dot{f}\end{array}$ & \begin{tabular}{|l|}
$\infty$ \\
$\infty$ \\
0 \\
$H$ \\
$\infty$ \\
$\dot{0}$ \\
$\varrho$
\end{tabular} & 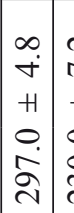 & $\begin{array}{l}\stackrel{1}{i} \\
+1 \\
\vdots \\
\dot{D} \\
\end{array}$ & $\begin{array}{l}\text { in } \\
n \\
+1 \\
0 \\
\dot{0} \\
\sim\end{array}$ & 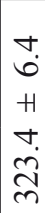 \\
\hline$\dot{0}$ & $\mid \begin{array}{l}\hat{b} \\
\infty \\
+ \\
+1 \\
n \\
\hat{8} \\
\dot{d}\end{array}$ & 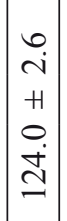 & $\mid \begin{array}{c}\vec{b} \\
+1 \\
0 \\
\dot{d} \\
\sigma\end{array}$ & $\mid \begin{array}{c}0 \\
\dot{0} \\
- \\
H \\
\infty \\
\dot{0} \\
\dot{\sigma}\end{array}$ & $\mid \begin{array}{c}- \\
\dot{ \pm} \\
+ \\
0 \\
\dot{a} \\
\tilde{n} \\
n\end{array}$ & 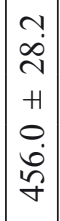 & $\begin{array}{l}\hat{\sigma} \\
+ \\
+ \\
\dot{0} \\
\dot{n} \\
n \\
i n\end{array}$ & $\begin{array}{c}a \\
i \\
+1 \\
n \\
\tilde{D} \\
0 \\
0\end{array}$ & $\begin{array}{l}\vec{n} \\
+1 \\
n \\
\tilde{n} \\
\tilde{n} \\
\hat{n}\end{array}$ & $\begin{array}{c}\infty \\
i \\
+1 \\
+1 \\
0 \\
\dot{0} \\
\tilde{n}\end{array}$ & $\left|\begin{array}{l}\vec{\infty} \\
+ \\
0 \\
0 \\
\dot{0} \\
\vec{N}\end{array}\right|$ & 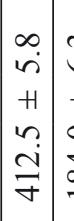 & $\begin{array}{l}n \\
\delta \\
H \\
0 \\
\dot{ \pm} \\
0 \\
-\end{array}$ & $\begin{array}{l}0 \\
\dot{r} \\
+1 \\
0 \\
\dot{j} \\
\dot{0} \\
-\end{array}$ & $\begin{array}{l}\vec{c} \\
\infty \\
+1 \\
0 \\
\dot{n} \\
\hat{\sim}\end{array}$ \\
\hline 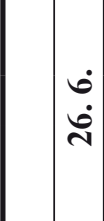 & $\mid \begin{array}{c}\vec{\infty} \\
\sim \\
n \\
+1 \\
n \\
\sim \\
\tilde{n} \\
a\end{array}$ & $\begin{array}{l} \\
\vec{i} \\
+ \\
0 \\
\dot{d} \\
\end{array}$ & $\mid \begin{array}{c}n \\
i n \\
+1 \\
\dot{j} \\
\dot{j} \\
\sim\end{array}$ & $\begin{array}{l}0 \\
\stackrel{.}{-} \\
+1 \\
0 \\
0 \\
\dot{\gamma} \\
\end{array}$ & $\mid \begin{array}{c}0 \\
\dot{n} \\
i \\
H \\
\infty \\
\dot{0} \\
\sim \\
\sim \\
\tilde{n}\end{array}$ & $\begin{array}{c}\sim \\
\tilde{n} \\
\tilde{n} \\
+1 \\
0 \\
\dot{0} \\
\dot{0} \\
\dot{+}\end{array}$ & $\begin{array}{c}2 \\
\infty \\
+ \\
+1 \\
+ \\
\dot{0} \\
\dot{2}\end{array}$ & $\begin{array}{c}\vec{a} \\
+1 \\
0 \\
\dot{0} \\
\ddot{0}\end{array}$ & $\begin{array}{l}\infty \\
i \\
+ \\
\infty \\
\dot{n} \\
\tilde{n}\end{array}$ & 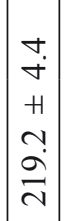 & $\left|\begin{array}{c}\infty \\
\dot{\sim} \\
H \\
0 \\
\stackrel{\dot{j}}{\sim} \\
\underset{\sim}{\sim}\end{array}\right|$ & 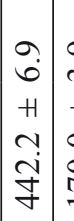 & $\begin{array}{l}\stackrel{a}{\dot{r}} \\
+1 \\
\dot{g} \\
\underline{I}\end{array}$ & $\begin{array}{l}n \\
+ \\
H \\
\infty \\
\dot{0} \\
\dot{+} \\
\sim\end{array}$ & $\begin{array}{l}m \\
n \\
H \\
0 \\
\dot{+} \\
\dot{n}\end{array}$ \\
\hline $\begin{array}{l}\dot{0} \\
\dot{0}\end{array}$ & $\mid \begin{array}{c}\hat{n} \\
\vec{j} \\
+1 \\
0 \\
\dot{0} \\
\dot{0}\end{array}$ & $\left|\begin{array}{c}n \\
i \\
+ \\
0 \\
\dot{j} \\
\dot{J}\end{array}\right|$ & $\mid \begin{array}{c}0 \\
\dot{n} \\
+1 \\
0 \\
\dot{+} \\
\dot{\sim}\end{array}$ & $\mid \begin{array}{l}0 \\
\dot{0} \\
+1 \\
\sim \\
\tilde{n} \\
\tilde{\lambda}\end{array}$ & $\mid \begin{array}{c}m \\
\infty \\
+1 \\
\infty \\
i \\
\tilde{m} \\
m\end{array}$ & 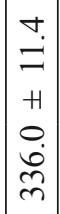 & $\begin{array}{l}\infty \\
\dot{n} \\
+1 \\
\sim \\
\tilde{n} \\
\tilde{n}\end{array}$ & $\mid \begin{array}{l}0 \\
\stackrel{2}{2} \\
+1 \\
n \\
\dot{2} \\
\stackrel{2}{2}\end{array}$ & $\begin{array}{c}\tilde{i} \\
i \\
H \\
0 \\
\stackrel{i}{\sim}\end{array}$ & $\begin{array}{l}0 \\
\dot{r} \\
+1 \\
0 \\
\dot{0} \\
\end{array}$ & $\begin{array}{l}q \\
\dot{+} \\
H \\
\dot{0} \\
\dot{\delta} \\
\infty\end{array} \mid$ & $\begin{array}{c}a \\
r \\
+1 \\
\sim \\
\tilde{b} \\
\infty \\
m\end{array}$ & $\begin{array}{l}m \\
i n \\
+1 \\
0 \\
\dot{n} \\
n\end{array}$ & 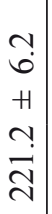 & $\begin{array}{l}\hat{\sigma} \\
+ \\
0 \\
\dot{0} \\
\hat{n}\end{array}$ \\
\hline $\begin{array}{l}\dot{2} \\
\vec{\sim}\end{array}$ & $\mid \begin{array}{c}n \\
\tilde{n} \\
+1 \\
+ \\
0 \\
\dot{0} \\
n \\
n\end{array}$ & $\left|\begin{array}{l}\hat{i} \\
+1 \\
m \\
\hat{b} \\
\underline{b}\end{array}\right|$ & 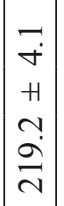 & $\mid \begin{array}{c}\infty \\
\dot{+} \\
+ \\
0 \\
\dot{0} \\
\dot{\sim} \\
\end{array}$ & $\mid \begin{array}{l}n \\
\dot{+} \\
+ \\
0 \\
\dot{0} \\
\stackrel{\sim}{\simeq}\end{array}$ & $\begin{array}{c}+ \\
\dot{n} \\
H \\
0 \\
\dot{n} \\
\tilde{n} \\
\sim\end{array}$ & $\begin{array}{l}\infty \\
\dot{+} \\
+ \\
0 \\
\dot{0} \\
\tilde{m} \\
m\end{array}$ & $\begin{array}{c}0 \\
\dot{i} \\
+ \\
+ \\
\tilde{j} \\
\tilde{y} \\
\dot{w}\end{array}$ & $\begin{array}{l}n \\
\dot{+} \\
+ \\
0 \\
\dot{j} \\
ٍ\end{array}$ & 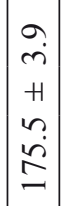 & $\begin{array}{l}n \\
\tilde{r} \\
H \\
\infty \\
\mathbb{I} \\
\tilde{I}\end{array}$ & 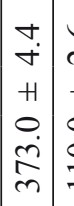 & $\begin{array}{l}0 \\
\dot{j} \\
+ \\
\dot{0} \\
\dot{0} \\
=\end{array}$ & $\begin{array}{l}n \\
i \\
+1 \\
0 \\
i \\
\infty \\
0\end{array}$ & 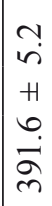 \\
\hline $\begin{array}{l}\dot{0} \\
\dot{I}\end{array}$ & {$\left[\begin{array}{c}\infty \\
\dot{c} \\
+ \\
\infty \\
\infty \\
\vec{\beth}\end{array}\right.$} & $\begin{array}{l}\hat{b} \\
0 \\
+ \\
\infty \\
\infty \\
\dot{f}\end{array}$ & & $\left|\begin{array}{c}a \\
\dot{+} \\
+1 \\
0 \\
\dot{\infty} \\
\infty\end{array}\right|$ & & 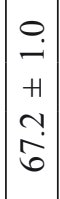 & 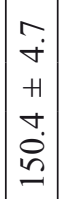 & $\begin{array}{l}0 \\
0 \\
+1 \\
0 \\
0 \\
\ddot{d} \\
\gamma\end{array}$ & $\begin{array}{c}\vec{v} \\
i \\
H \\
n \\
\tilde{n} \\
i n\end{array}$ & $\begin{array}{c}\tilde{n} \\
i \\
H \\
n \\
\tilde{g}\end{array}$ & $\left|\begin{array}{l}\infty \\
i \\
H \\
0 \\
i \\
i\end{array}\right|$ & 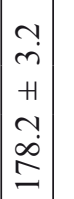 & & $\begin{array}{l}\hat{i} \\
+ \\
\dot{b} \\
\dot{\infty}\end{array}$ & $\begin{array}{l}\vec{r} \\
+1 \\
0 \\
0 \\
\infty \\
\tilde{n}\end{array}$ \\
\hline $\begin{array}{l}\frac{\mathscr{U}}{\mathscr{U}} \\
\text { के }\end{array}$ & 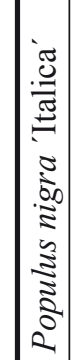 & 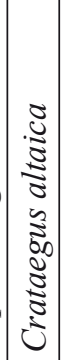 & 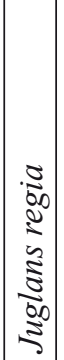 & $\mid$ & 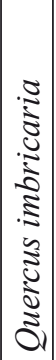 & 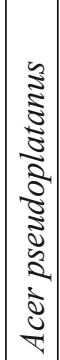 & 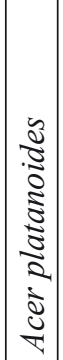 & 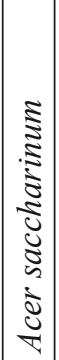 & 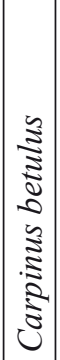 & 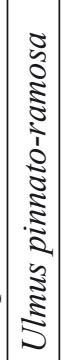 & 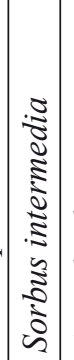 & 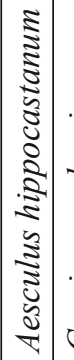 & $\begin{array}{c}c \\
0 \\
0 \\
0 \\
0 \\
0 \\
0 \\
0 \\
0 \\
0\end{array}$ & $\begin{array}{c}0 \\
0 \\
\vdots \\
0 \\
0 \\
0 \\
\vdots \\
\vdots \\
0 \\
0 \\
2\end{array}$ & 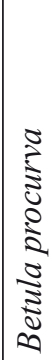 \\
\hline
\end{tabular}




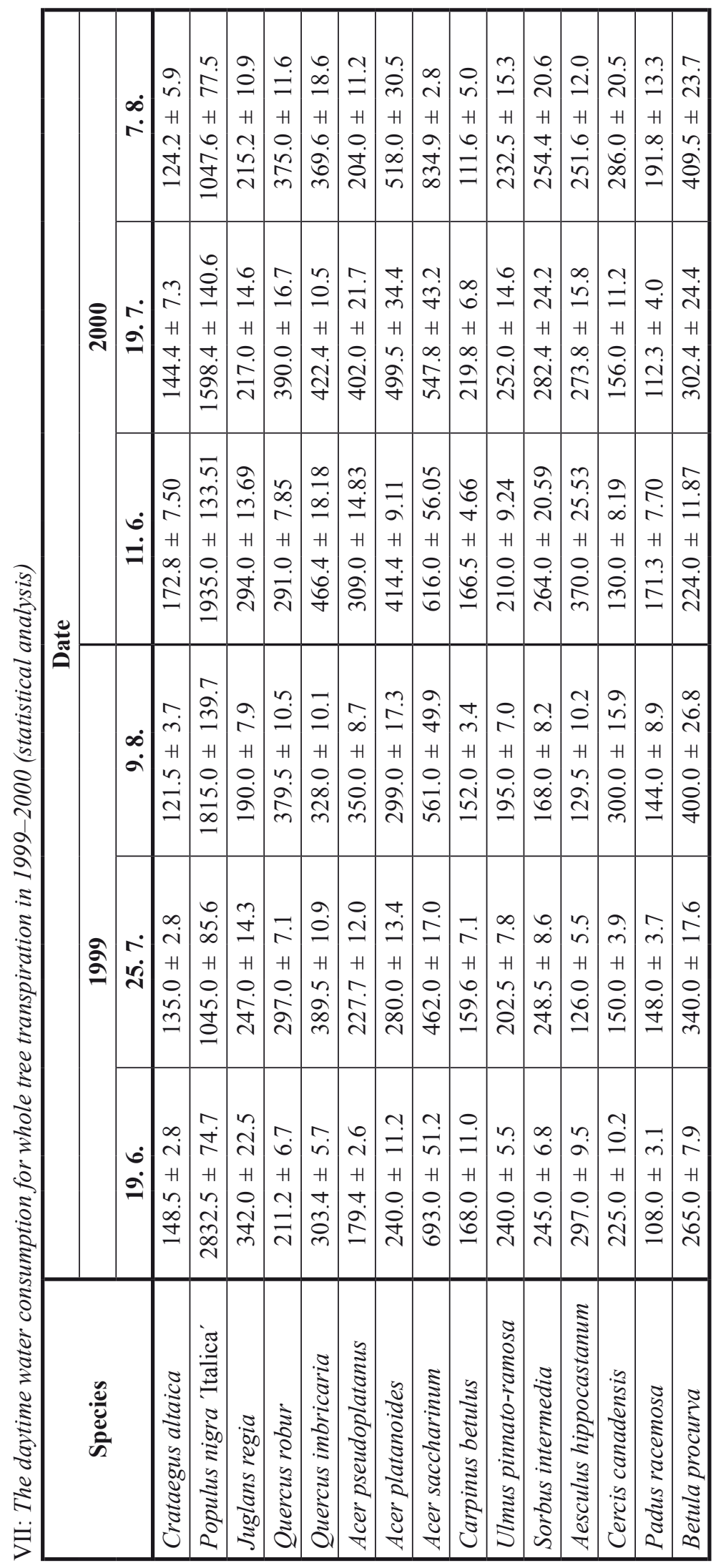


m

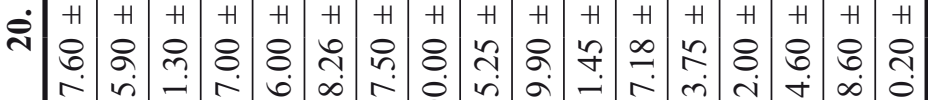
స்

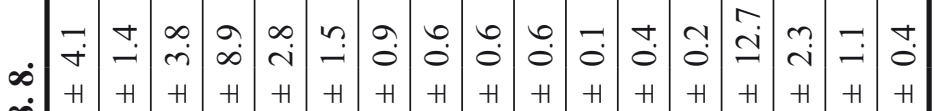

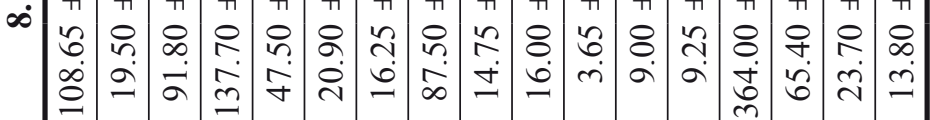

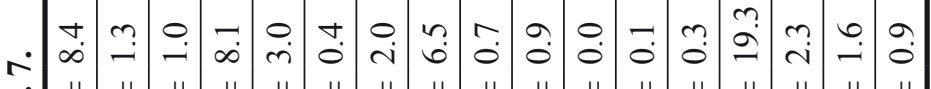

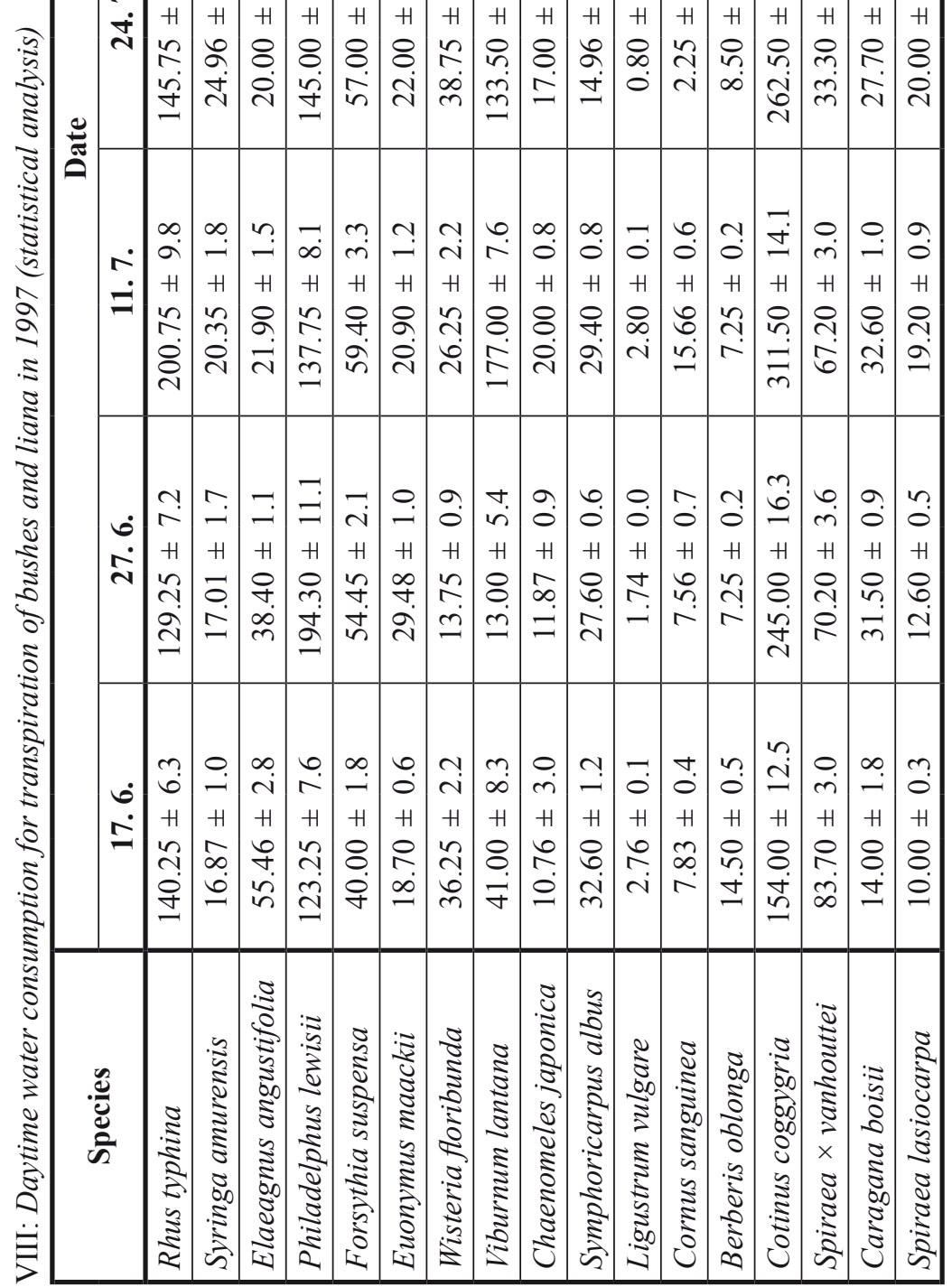




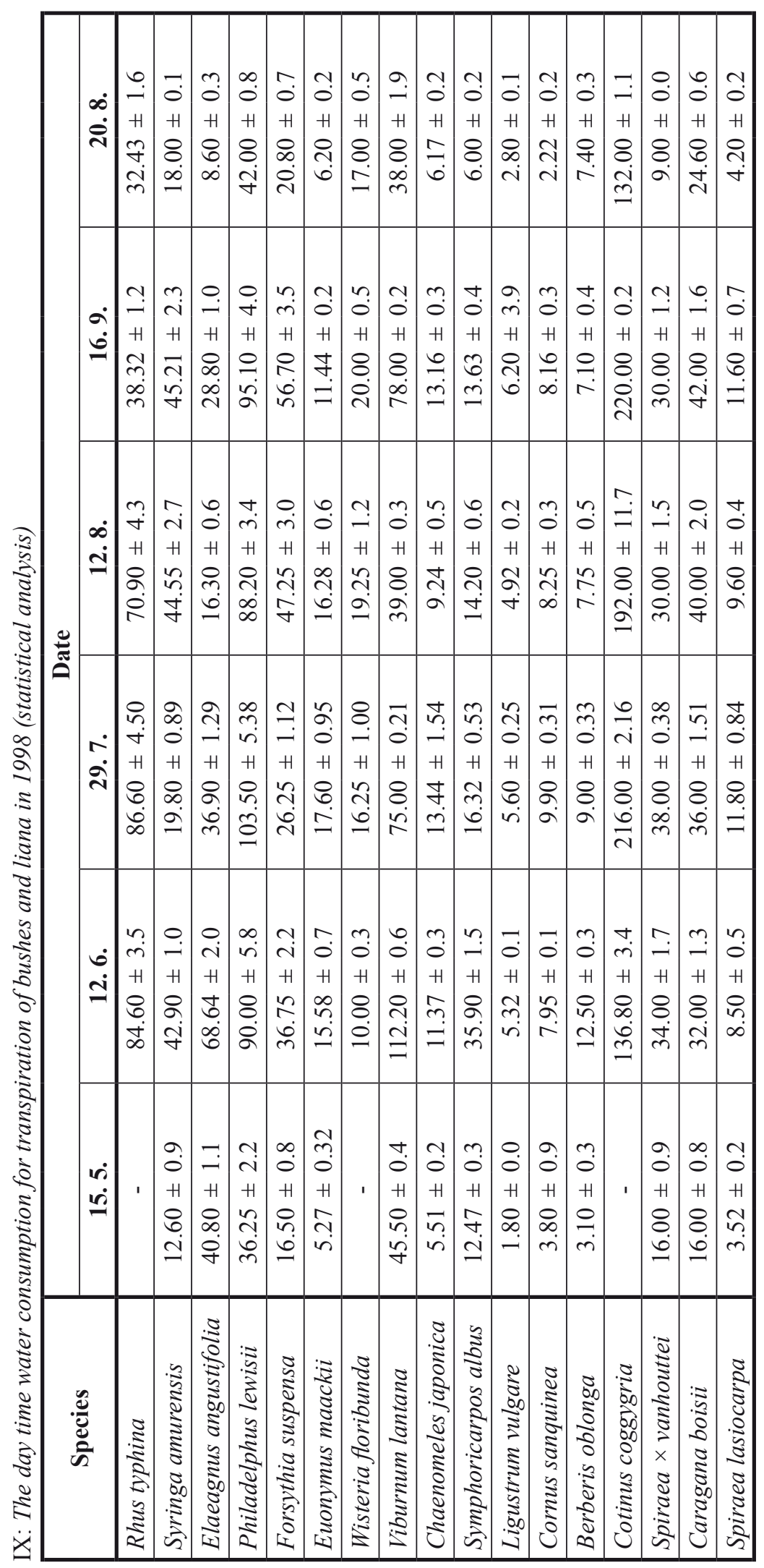


X: The daytime bush and liana water consumption for transpiration in 1999 (statistical analysis)

\begin{tabular}{|l|r|r|r|r|}
\hline \multirow{2}{*}{ Species } & \multicolumn{5}{c|}{ Date } \\
\cline { 2 - 5 } & $\mathbf{2 1 . 6 .}$ & $\mathbf{2 6 . 7 .}$ & $\mathbf{1 8 . 8 .}$ & $\mathbf{1 2 . 9 .}$ \\
\hline Rhus typhina & $63.45 \pm 1.4$ & $63.45 \pm 1.9$ & $23.50 \pm 0.6$ & $27.90 \pm 0.6$ \\
\hline Syringa amurensis & $26.55 \pm 0.3$ & $39.00 \pm 0.7$ & $45.00 \pm 1.1$ & $25.98 \pm 0.8$ \\
\hline Elaeagnus angustifolia & $14.00 \pm 0.4$ & $12.00 \pm 0.2$ & $50.00 \pm 1.9$ & $12.00 \pm 0.4$ \\
\hline Philadelphus lewisii & $117.00 \pm 7.9$ & $92.80 \pm 4.0$ & $88.00 \pm 4.2$ & $56.70 \pm 3.1$ \\
\hline Forsythia suspensa & $25.60 \pm 0.7$ & $39.20 \pm 0.5$ & $96.20 \pm 2.5$ & $31.50 \pm 1.7$ \\
\hline Euonymus maackii & $18.00 \pm 0.8$ & $15.50 \pm 0.8$ & $11.85 \pm 0.6$ & $9.58 \pm 0.6$ \\
\hline Wisteria floribunda & $16.25 \pm 0.9$ & $18.50 \pm 0.7$ & $15.00 \pm 0.9$ & $16.00 \pm 0.4$ \\
\hline Viburnum lantana & $102.00 \pm 2.4$ & $49.20 \pm 4.6$ & $78.00 \pm 2.5$ & $58.80 \pm 4.5$ \\
\hline Chaenomeles japonica & $9.60 \pm 0.6$ & $7.90 \pm 0.6$ & $9.37 \pm 0.4$ & $8.00 \pm 0.9$ \\
\hline Symphoricarpos albus & $16.38 \pm 0.5$ & $7.78 \pm 0.6$ & $7.80 \pm 0.3$ & $6.20 \pm 1.0$ \\
\hline Ligustrum vulgare & $4.40 \pm 0.2$ & $3.44 \pm 0.4$ & $4.72 \pm 0.2$ & $2.72 \pm 0.4$ \\
\hline Cornus sanquinea & $6.90 \pm 0.1$ & $5.16 \pm 0.6$ & $8.10 \pm 0.3$ & $3.20 \pm 0.3$ \\
\hline Berberis oblonga & $9.13 \pm 0.8$ & $6.30 \pm 0.5$ & $9.57 \pm 0.8$ & $6.75 \pm 0.4$ \\
\hline Cotinus coggygria & $133.00 \pm 5.9$ & $157.50 \pm 10.0$ & $179.20 \pm 1.6$ & $145.60 \pm 6.2$ \\
\hline Spiraea $\times$ vanhouttei & $34.40 \pm 0.6$ & $45.00 \pm 1.3$ & $59.00 \pm 2.4$ & $27.00 \pm 1.3$ \\
\hline Caragana boisii & $26.00 \pm 1.3$ & $44.00 \pm 2.3$ & $35.00 \pm 1.8$ & $28.00 \pm 1.7$ \\
\hline Spiraea lasiocarpa & $11.50 \pm 0.5$ & $15.00 \pm 0.8$ & $14.50 \pm 0.9$ & $8.20 \pm 0.7$ \\
\hline
\end{tabular}

\section{SUMMARY}

From the all investigated tree species, Populus nigra L. 'Italica' differs by its high values of the daytime water consumption for transpiration. It can transpired up to $2832.5 \mathrm{~kg}$ day $^{-1}$ of water from the total leaf surface. Acer saccharinum L. can, in certain days, evaporate more than $1000 \mathrm{~kg}$ of water per day. These are two examples where a high value of transpiration and also the large leaf surface are responsible for this results. According to the indications of the day water consumption for transpiration, Acer pseudoplatanus L., Acer platanoides L., Quercus robur L., Quercus imbricaria Michx., Betula procurva Litv., Sorbus intermedia (Ehrh.) Pers., Cercis canadensis L., Juglans regia L., Ulmus pinnato-ramosa Dieck. ex Kochne. and Aesculus hippocastanum L. reaches a medium values - from 126.0 up to $518.0 \mathrm{~kg}$.day ${ }^{-1}$.

A minimum amount of water per day is transpired by Padus racemosa (L.). Gillib. and Crataegus altaica Lge. Among bushes the biggest amount of water,

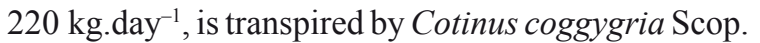

By high parameters of the day time charge of water consumption for transpiration are characterized also Rhus typhina L., Viburnum lantana L., Philadelphus lewisii Pursh., Elaeagnus angustifolia L., Syringa amurensis Rupr., Forsythia suspensa (Thunb.) Vahl., Spiraea $\times$ vanhouttei (Briot). Zab. and Caragana boisii Schneid. Smaller amount of water is transpired by Ligustrum vulgare L. and Cornus sanquinea L. The received data about the whole wood plant daytime water consumption for transpiration can help to determine a degree of monitored woody plant species influence on air humidifying and consequently also on a various settlements microclimate character. Besides, in accordance with results of our research, it is possible to recommend some trees and bushes, distinguished by various amount of daily delivered water by transpiration, for planting at the sites with various water supply. Rational application of woody plantings, according to their ability to save precipitation, is an important factor enable to influence our environment. 


\begin{abstract}
SOUHRN
Denní průběh transpiračního toku dřevin

Údaje o denním výdeji vody dřevin výparem umožňují stanovení stupně vlivu jednotlivých druhů dřevin na relativní vlhkost vzduchu a celkové mikroklima porostu. To následně umožňuje lepší využití dřevin na různých typech stanovišst’ a v rámci sídel.

$\mathrm{K}$ experimentům byly vybrány introdukované druhy listnatých dřevin, pěstované $\mathrm{v}$ Botanické zahradě Národní Akademie věd Kyrgyzské republiky v Biškeku. Z celkového počtu 32 testovaných druhů se jednalo o 15 druhů stromů, 16 druhů keřů a 1 druh pnoucí dřeviny. Intenzita transpirace byla měřena polními transpirometry modifikovanou metodou Špota (1992). Mimo jiné parametry byla sledována také plocha jednotlivých listů, celková listová plocha dřevin a dynamika změn v průběhu vegetace.

Nejvyššími hodnotami denního výdeje vody transpirací se vyznačoval Populus nigra L. 'Italica', který může za den vydat až 2832,5 kg vody (vztaženo k celkové listové ploše). Více než $1000 \mathrm{~kg}$ vody za den může odpařit Acer saccharinum L. Jsou to dva prŕklady, ve kterých lze pozorovat významný vliv transpirace ve vztahu k celkové listové ploše. Středních hodnot v ukazateli denního výdeje vody transpirací bylo dosaženo u Acer pseudoplatanus L., Acer platanoides L., Quercus robur L., Quercus imbricaria Michx., Betula procurva Litv., Sorbus intermedia (Ehrh.) Pers., Cercis canadensis L., Juglans regia L., Ulmus pinnato-ramosa Dieck. ex Kochne. a Aesculus hippocastanum L. U těchto druhů dřevin se naměřené hodnoty pohybovaly v rozmezí od 126 do $518 \mathrm{~kg}$ za den. Minimální množství vody za den transpirují Padus racemosa (L.). Gillib. a Crataegus altaica Lge.

U keřů byl zjištěn nejvyšší výdej vody u Cotinus coggygria Scop. (220 kg za den). Relativně vysoký výdej vody charakterizuje také následující druhy dřevin: Rhus typhina L., Viburnum lantana L., Philadelphus lewisii Pursh., Elaeagnus angustifolia L., Syringa amurensis Rupr., Forsythia suspensa (Thunb.) Vahl., Spiraea $\times$ vanhouttei (Briot.) Zab. a Caragana boisii Schneid. Malé množství vody vypařují Ligustrum vulgare L. a Cornus sanquinea L.

Závěry provedených experimentálních měření umožní lepší specifikaci nároků testovaných introdukovaných dřevin a umožní přesnější zařazení do skupin, charakterizujících vhodnost dřevin pro trvalé výsadby na plochy s rozdílným vodním režimem. Realizace výsadeb zeleně, zohledňující nároky stromů a keřů na dodatkovou závlahu při následné údržbě, umožní výrazně snížit náklady na závlahovou vodu. V podmínkách klimatu Kyrgyzstánu (semiaridní a aridní oblast) se jedná o velmi důležitý faktor, zejména v horkém letním období.
\end{abstract}

dřeviny, transpirace, mikroklima, výdej vody

\section{REFERENCES}

ANASTASEV, N. M., HARAHINOV, M. K.: Questions of microclimate and out organization of public services of populated places, Moscow, 1936, $89 \mathrm{p}$.

AKHMATOV, M. K., OSKONBAEVA, R. K., SHPOTA, L. A.: The new method of using field transpirometers on determining intensive transpiration of shrubs and lianas. Introduction and acclimatization of plants in Kyrgyzstan. Bishkek: Ilim, 1999, pp. 120-123, ISBN 5-8355-1040-3

DOSPECHOV, B.A.: Metodika polevogo opyta sosnovami statističeskoj obrabotki rezultatov issledovanij. Moskva, Kolos, 1976, 416 p.

CHISTJAKOVA, S. B., SEMENOVA, E. S.: The settlement garden problems of populated points with the questions of microclimate, Moscow, 1962, $102 \mathrm{p}$.
IZDESBSKIJ, A. M.: Experiment of physic-hygienic studying of green plantations microclimate. Hygiene and sanitary, 1949, 5, pp. 34-37

KOCHKUMBAEV, T. A., ESENALIEVA, S. S., AKHMATOV, M. K., SHPOTA, L. A., OSKONBAEVA, R. K.: To the definition method of the leaves areas of woody plants, Introduction and acclimatization of plants in Kyrgyzstan. Bishkek, 1999, pp. 132-134, ISBN 5-8355-1040-3

KOCHKUMBAEV, T. A., ESENALIEVA, S. S., AHMATOV, M. K., SHPOTA, L. A.: To the definition method of the leaves areas and general leaves surface of woody plants. $7^{\text {th }}$ International Conference ,International Meeting of Young Scientists in Horticulture", Lednice, 1999, pp. 322323, ISBN 80-7157-384-1, 425 p.

KOWASHIMA, S.: Effect of vegetation on surface temperature in urban and suburban areas in winter. 4th Int. Conf. "Urban Climate, Plann. and Build", 
Kyoto, 1989, In.: Energy and Build., 1990, Vol. 15., 3-4, pp. 465-469.

KUZMINA, T. F.: Planning, building and settlement garden of city streets in Central Asia conditions. Hygiene and sanitary, 1970, 11, pp. 11-12

LIBBERT, E.: Physiology of plants, Moscow, 1976, $580 \mathrm{p}$.

LUNCE, L. B.: Urban settlement garden, Moscow, 1974, $275 \mathrm{p}$.

MASHINSKYJ, L. O.: Some questions of settlement garden (record typed from shorthand of the lecture), Moscow, 1962, 5 p.

NESTEROV, V. G.: General forestry, Moscow, 1949, $135 \mathrm{p}$.

POPOV, S. P., JAKOVLEVA, N. V.: Poplar is the tree of future, Echo of the sciences, Information of NAS KR, 1996, 2, pp. 98-99, ISSN 0002-3221

RAZA, S. H., MURTHY, M. S. R. et al.: Effect of vegetation on urban climate and healthy urban colonies. 4th Int. Conf. "Urban Climate, Plann. and Build", Kyolo, 1989, In.: Energy and Build., 1990, Vol. 15, 3-4, pp. 487-491.

SHPOTA L. A.: The field methods and equipments for physiological control of plant conditions in field and natural condition of growing. Bishkek: Ilim, 1992, 154 p., ISBN 5-8355-0489-6

SAITO, I., ISHIHARA, O., A KATAYAMA, T.: Study of the effect of green areas on the thermal environment in an urban area. 4th Int. Conf. "Urban Climate, Plann. and Build", Kyoto, 1989, In.: Energy and Build., 1990, Vol. 15, 3 - 4, pp. 493498.

VILLY, K., DETEV, B.: Biology, Moscow, 1974, 821 p.

WILMERS, F.: Effect of vegetation on urban climate and building. 4th Int. Conf. "Urban Climate, Plann. and Build", Kyoto, 1989, In.: Energy and Build., 1990, Vol. 15, 3 - 4, pp. 507-514

Akhmatov Medet Kenjebaevich, Ph.D., Botanical Garden of National Academy of Sciences of Kyrgyz Republic, 720064 Bishkek city, Akhunbaeva street 1a., Kyrgyz Republic, Doc. Dr. Ing. Petr Salaš, Ústav šlechtění a množení zahradnických rostlin, Mendelova zemědělská a lesnická univerzita v Brně, Valtická 337, 69144 Lednice, Česká republika 
